\title{
Spinal tuberculosis
}

\author{
J. W. JACKSON \\ Royal National Orthopaedic Hospital, Stanmore, Middlesex
}

\begin{abstract}
Summary
Seventy-one out of eighty-four cases of spinal infection operated on at the Royal National Orthopaedic Hospital in the last 12 years were due to tuberculosis. The clinical features are reviewed.
\end{abstract}

NeARLy 200 patients with spinal tuberculosis have been admitted to the Royal National Orthopaedic Hospital at Stanmore during the past 10 years. The yearly numbers varied between ten in 1963 and thirty in 1968 and so far there has been no discernible fall. This may be due to an increase in the number of immigrants in our population, or to cases being referred from a wider area or simply to the fact that a fixed number of beds can only admit a fixed number of cases.

Not all of these patients are brought forward for surgical treatment. There is no rigid scheme of selection into those which should be treated medically or surgically. Bed rest, which usually involves immobilization in a plaster bed, and triple drug chemotherapy is still the primary method of treatment and surgery but an incident in this regimen.

Naturally, where there is a limited lesion with minimal deformity and little bone destruction or abscess formation medical treatment is the method of choice, but even now, in the 1970s, an apparently minor lesion may progress to abscess formation in spite of adequate bed rest and chemotherapy.

On the other hand, where a number of vertebrae are diseased leading to an unstable spine or considerable deformity, possibly with paravertebral or psoas abscess formation and spinal cord involvement, surgical decompression, with the removal of all pus and caseous disease, dead bone and necrotic disc followed by spinal fusion is imperative.

In between are those cases in which there is doubt about the actual diagnosis-where the history is unusual or the presentation atypical, we have preferred to obtain bacteriological or histological confirmation of the diagnosis from material obtained at operation before submitting any patient to a prolonged period of immobilization and anti-tuberculous chemotherapy. This is of particular importance where bone destruction is due to tumour-in this case a myeloma.

Of eighty-four spinal infections operated on over the past 12 years, seventy-one were tuberculous and thirteen due to other organisms. One man, early in the series, had received intermittent courses of antituberculous chemotherapy over a period of 7 years. He had a staphylococcal osteomyelitis due to septicaemia following a septic varicose vein ligation. His X-ray showed a well defined paravertebral abscess but an atypical vertebrae lesion.

I do not wish to discuss the differential diagnosis of the two types of lesion.

Backache was the presenting symptom in every case, sometimes trivial and often intermittent so that its significance was ignored and palliative treatment prescribed.

On average the initial symptoms were present for 5 months although in tuberculosis the disease may have been present for up to 40 years. The sex ratio is the same in each group: $M: F=2: 1$. Fifty per cent of those with tuberculosis were born in England, 20\% were Irish and the remaining 30\% were from India, Africa, Jamaica or the Mediterranean. There were no true immigrants in the nontuberculous osteomyelitis series as the one school teacher from Pakistan was sent over to this country specially for treatment. The tuberculous cases are widely distributed throughout each decade with the maximum incidence below the age of 50 . None of the osteomyelitis cases were below the age of 30 .

Fifty of the tuberculous cases had other evidence of this disease. In three there was active lung disease with a positive sputum in two of them. In five the pleura was involved by direct extension of the spinal disease, twelve had genito-urinary disease and positive urine cultures for acid-fast bacilli were obtained in five of them. All patients with persistent pyuria had intravenous pyelography and urine cultures for tubercle bacilli carried out. Two patients had had nephrectomy for renal tuberculosis and one man died from renal failure 5 years after operation on his spine. Others showed the usual evidence of calcified glands in the neck, chest or abdomen. In five the spinal column was involved at more than one level and seven others had other bone or joint disease. There was one tuberculous thyroid abscess and one ischiorectal abscess.

In the other series the only evidence of tuberculosis was a healed calcified lesion at the apex of the right lung in a man of 60 . More important in this group is a history of previous sepsis, two had septi- 
caemia and three urinary infections associated with prostatic enlargement for which surgical treatment had been carried out in the preceding 6 months.

The sedimentation rate is of no diagnostic value but is a useful index of the response to treatment and a warning of reactivation. The Mantoux reaction was negative in one of the osteomyelitis cases and the staphylococcal antibody titres elevated in three.

Kyphosis is not very marked in non-tuberculous osteomyelitis and paraplegia never part of the initial symptom-complex. It was a late development in sixteen cases of longstanding tuberculous disease. In five, weakness or sensory impairment were present from the onset. Paraplegia was rapid and complete in two cases, one in each series. Their spinal deformity was not severe and it is thought that the blood supply to the spinal cord had become impaired because of the rapid onset and the fact that no recovery took place. They account for the only deaths in hospital.

Unless it is possible to isolate an organism from a sample of pus from an abscess or skin sinus radiology is the most helpful diagnostic investigation.

There is little difference in the level of the spinal lesion in each series. The ninth dorsal vertebra is the most frequent level for the disease in tuberculosis and the incidence tapers off from here to the lumbar and cervical spine. In osteomyelitis the maximum incidence is at the level of D7. The cervical vertebra were not involved in any case and the lumbar spine (L1 and 2) in only one patient.

Narrowing of an intervertebral disc is usually the earliest sign in tuberculosis and is followed by rarefaction of the bodies adjacent to the disc.

In osteomyelitis the disc space is intact or apparently expanded and the bodies show sclerosis on either side of it. The anterior margins of the bodies tend to be preserved whereas in tuberculosis the disease spreads up and down under the anterior longitudinal ligament and the anterior margins of the bodies become eroded and excavated as if by an aneurysm. Occasionally there is central excavation of a body or similar excavation posteriorly which may at the same time erode the pedicle. Sequestrum function is sometimes seen and confirmed at operation.

Other cases show a dense bony plate in a vertebra between the disease and an adjacent disc; presumably this is avascular necrosis. These changes are not seen in osteomyelitis nor is the deformity ever so severe probably because no more than two vertebrae are ever involved. If disease is present at two levels then it is almost certainly tuberculosis, particularly when the same case has a bilateral calcified psoas abscess. Paravertebral abscess was equally common in each series and the incidence was not any higher in the immigrant population. All the non-tuberculous paravertebral abscesses were staphylococcal.

There were seventy-one cases in the 'tuberculous' series and in sixty-one of these tubercle bacilli were identified or satisfactory histological evidence of tuberculosis obtained from operation material. In five, in which confirmation was not obtained, the disease had been present for between 5 and 40 years. There was little clinical or radiological doubt in three and in two an open diagnosis is maintained 2 years after operation.

In the thirteen 'other infection' cases organismo were isolated in seven: Staph. aureus, five; Esch. colis one; Proteus, one. In three the histology reported evidence of chronic inflammation and in three the findings were inconclusive.

Most of these patients, at the time of operatior get an iliac crest bone graft to the vertebral bodies an this is followed by a posterior spinal fusion if three or more bodies are involved by the disease. They are nursed on a plaster bed with an anterior turning cast for 3 months after operation; after this they are fitted with a spinal brace which is worn for 3 months or longer if necessary.

\section{Acknowledgment}

This talk is in part derived from a Hunterian Lecture delivered at the Royal College of Surgeons on 10 June 1970 and published in the Annals of the Royal College of Surgeons of England, Vol. 48, Feb. 71, 83-98. 